Case Report

\title{
A COMPLEX CASE OF HAEMOGLOBIN E DISEASE WITH IMMUNE THROMBOCYTOPENIA AND COMBINED IRON, FOLATE AND VIT $B_{12}$ DEFICIENCY
}

\author{
Md Sirazul Islam ${ }^{1}$, Tashmim Farhana Dipta ${ }^{2}$ and Gazi Sharmin Sultana ${ }^{1}$ \\ ${ }^{1}$ Department of Clinical Pathology, Clinical Biochemistry \& Haematology, Bangladesh Institute of \\ Research \& Rehabilitation in Diabetes Endocrine and Metabolic Disorders (BIRDEM), Dhaka \\ ${ }^{2}$ Department of Tranfusion Medicine, BIRDEM and Ibrahim Medical College, Dhaka
}

\begin{abstract}
This is a case report of a 13 years old indigenous 'Garo' girl who presented with purpuric spots and ecchymotic patches all over the body with menorrhagia, mild jaundice, severe anaemia, marked thrombocytopenia, moderate neutrophil leucocytosis and reticulocytosis. Investigations revealed this as a complex case of Haemoglobin E disease with immune thrombocytopenia (ITP) and combined iron, folate and vitamin $B_{12}$ deficiency. The case is discussed thoroughly.
\end{abstract}

Ibrahim Med. Coll. J. 2012; 6(1): 31-33

Key words: $\mathrm{Hb} \mathrm{E}$ disease, immune thrombocytopenia, combined deficiency.

\section{Cases report}

A 13 years old, indigenous 'Garo' girl hailing from Haluaghat, Mymensingh was admitted in the Paediatric ward of BIRDEM in June 2010 with the complaints of reddish black rashes all over the body for previous two months associated with excessive vaginal bleeding during previous two menstrual cycles with periods of about ten days each and similar excessive bleeding during current menstruation for last five days and gum bleeding for seven days. Initially she got three units of blood transfusion but her condition did not improve much. She had no history of vomiting, convulsion, photophobia or significant drug ingestion.

On examination the girl was severely anaemic and mildly icteric with normal vital signs. Multiple petechiae, purpura and bruise were found. There was no lymphadenopathy, organomegally, bony tenderness or signs of meningeal irritation. All other systemic examinations revealed no abnormality. Initial blood count reports showed hemoglobin $5.7 \mathrm{~g} / \mathrm{dl}, \mathrm{MCV}: 66.5$ fl, RDW (CV): $27.3 \%$, total leukocyte count (WBC): $11,900 /$ cmm \& differential count: N-76\%, L-19\%, M-04\%, E-01\%, B-00\% and platelet count: 5000/cmm.
Reticulocyte count was $10 \%$. Blood film showed dimorphic picture with anisochromia and anisopoikilocytosis, some hypochromic microcytes and normochromic normocytes, a few macrocytes including macro-ovalocytes, target cells, elliptocytes, irregularly contracted cells and few fragmented cells including schistocytes and helmet cells, a few polychromatic cells and occasional nucleated red cells. WBCs were mature with mildly increased Neutrophils. Platelets were markedly decreased in number with normal morphology (Figure-1). The features were nonspecific with neutrophil leucocytosis and thrombocytopenia but possibilities to be ruled out were- Evans's syndrome i.e., autoimmune haemolytic anemia with immune thrombocytopenia, haemoglobinopathy, microangiopathic haemolytic anaemia with disseminated intravascular coagulation and combined iron and folate / vit $\mathrm{B}_{12}$ deficiency. Bone marrow examination revealed a moderately hypercellular particulate marrow with decreased M/E ratio (about 1:2). Erythropoiesis was markedly hyperactive and mainly normoblastic with some megaloblastic features.

Address for Correspondence:

Professor Md Sirazul Islam, Department of Clinical Pathology, Clinical Biochemistry \& Haematology,

122 Kazi Nazrul Islam Avenue, BIRDEM Dhaka-1000, Email: drmsiraz@gmail.com 

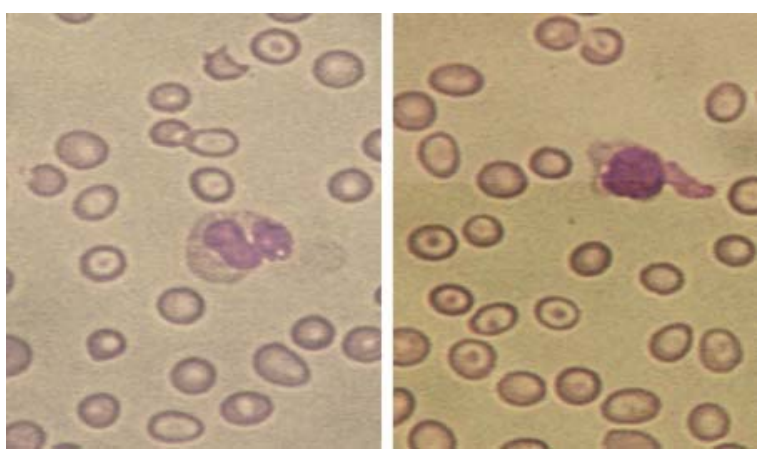

Fig-1. Blood Film showing dimorphic picture with anisopoikilocytosis, target cells, macro-ovalocytes, schistocytes and marked thrombocytopenia.

Granulopoiesis appeared normal with maturing to segmented forms. Giant metamyelocytes were not seen. Blasts were not increased. Megakaryocytes appeared normal in number and morphology with occasional hyperpolyploid forms (Figure-2). Perls' staining for iron revealed no stainable marrow iron. In conclusion, bone marrow revealed marked erythroid hyperplasia with some megaloblastic changes and absent stainable marrow iron, suggestive of combined iron and folate/ Vit $\mathrm{B}_{12}$ deficiency. The features were also compatible with ITP as well due to the presence of adequate number of megakaryocytes.

Other investigations showed: blood group - 'A' $\mathrm{Rh}$ (D) positive, prothrombin time (PT) -11.4 sec (control 12.0 sec), APTT-24.5sec (control $28.0 \mathrm{sec}$ ), fibrinogen degradation product (FDP) - negative, serum iron: 6.8 $\mu \mathrm{mol} / \mathrm{l}$ (Female ref. range 6.6-26.0), total iron binding capacity (TIBC): $77.8 \mu \mathrm{mol} / \mathrm{l}$ (Ref. range: 40.8-76.6), serum ferritin: $12 \mathrm{ng} / \mathrm{ml}$ (Female ref. range:15-

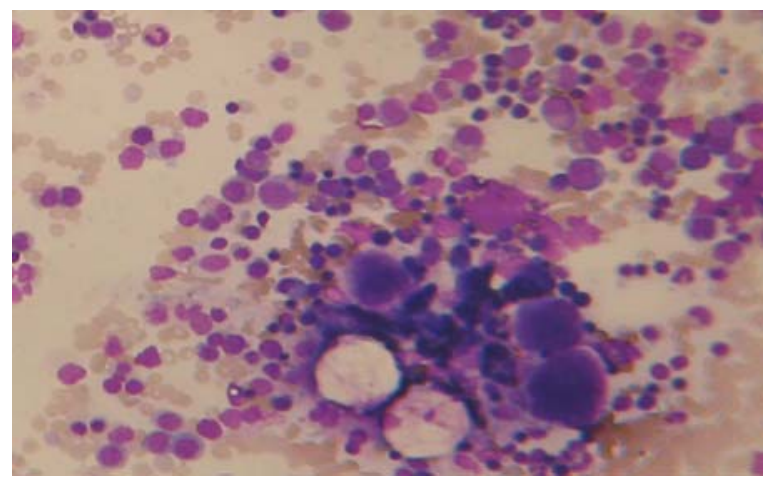

Fig-2. Bone marrow smear showing erythroid hyperplasia with some megaloblastic changes and normal number of megakaryocytes.
150), Vit. $B_{12}-186 \mathrm{pg} / \mathrm{ml}$ (Ref. range: $208-963 \mathrm{pg} / \mathrm{ml}$ ), folate-6.0ng/ml (Ref. range: $7.2-15.4 \mathrm{ng} / \mathrm{ml}$ ). Urine $\mathrm{R} / \mathrm{M} / \mathrm{E}$ was normal with no haemoglobinuria, SGPT, SGOT and alkaline phosphatases were normal.

Hb-electrophoresis showed HbA: $28.4 \%$, Hb E: $71.6 \%$ on $1 \%$ agarose gel at alkaline $\mathrm{pH}$ (8.6) suggesting a diagnosis of homozygous $\mathrm{HbE}$ (EE) disease. Parent's haemoglobin electrophoresis revealed both parents as haemoglobin E traits. Serum bilirubin (total) $-3.2 \mathrm{mg} /$ $\mathrm{dl}$, mostly indirect $(3.0 \mathrm{mg} / \mathrm{dl})$, direct and indirect coomb's tests were negative, antinuclear antibody (ANA) screening was negative, serum calcium was normal. Our final diagnosis was haemoglobin E disease with ITP and combined iron, folate and vitamin $B_{12}$ deficiency.

The patient was managed with prednisolone. Her platelet count improved after four days of starting treatment and her menstrual bleeding also stopped. She was also given supplementation of iron, folate and vitamin $B_{12}$. Tapering of prednisolone was done after 15 days when her platelet count became $210000 / \mathrm{cmm}$ and the patient was asymptomatic with fading and regression of skin lesions.

\section{Discussion}

The case aroused lot of suspicions like haemoglobinopathy, microangiopathic haemolytic anaemia with disseminated intravascular coagulation and combined iron and folate/vit $\mathrm{B}_{12}$ deficiency due to complex peripheral blood findings. Appropriate investigations finally revealed that it was a complex case of haemoglobin E disease with ITP and co-existent combined iron, folate and vitamin $\mathrm{B}_{12}$ deficiency.

Haemoglobinopathies usually producing iron overload may at times co-exist with iron deficiency further aggravating already present microcytic anaemia. Heterozygous carriers and homozygous Hb-EE cases may not uncommonly present with iron deficiency when there is minimum or no hemolysis. On the other hand, homozygous disease conditions commonly producing iron overload may rarely produce iron deficiency as well. The scenario may further be complicated with ITP and folate or vitamin $B_{12}$ deficiency. However, there is no correlation between Hb-E disease and ITP, probably it is a coincidental associated pathology. Thalassaemia and haemoglobinopathies are autosomal recessive inherited disorders. Hemoglobin $(\mathrm{Hb}) \mathrm{E}$ is 
one of the world's most common and important mutations. ${ }^{1} \mathrm{Hb} \mathrm{E}$ trait and $\mathrm{Hb} \mathrm{EE}$ are usually mild disorders whose phenotype ranges from asymptomatic to severe. The most common presentation of $\mathrm{Hb} \mathrm{E}$ disease is no or mild anaemia, jaundice, fever, abdominal pain and gastrointestinal disturbance with or without any evidence of haemolysis. ${ }^{2}$ Bone marrow frequently shows mild erythroid hyperplasia. On electrophoresis $\mathrm{Hb} \mathrm{E}$ always predominates in $\mathrm{Hb} \mathrm{E}$ disease. The amount of $\mathrm{Hb} \mathrm{E}$ is $87.7 \% \pm 5.90$ in homozygous $\mathrm{Hb} \mathrm{E} .^{3}$ As our case got multiple transfusion so her $\mathrm{Hb} \mathrm{A}$ was increased and $\mathrm{HbE}$ was slightly reduced by the dilution effect of transfused normal blood.

ITP is a relatively common disorder and highest incidence has been considered to be in the women aged 15 -50 years. It may be acute (childhood ITP) and chronic (adult ITP). The clinical features of ITP include abrupt or gradual onset of symptoms, purpura, menorrhagia, epistaxis, gingival bleeding. ITP is the most common cause of acute onset of thrombocytopenia in otherwise healthy child. In ITP, only platelet count is decreased but haemoglobin and leukocyte counts remain almost normal unless profuse bleeding occurs. Bone marrow usually reveals increased or normal number of megakaryocytes with some young forms. In our case instead of increase in young forms occasional hyperpolyploid megakaryocytes were present. This is probably due to co-existent folate and vitamin B12 deficiency. Granulopoiesis may be normal with some degree of marrow eosinophilia.

Initially, due to increased reticulocyte count at presentation our case was confused with Evans's syndrome. Evans's syndrome or auto-immune haemolytic anaemia with ITP was excluded as the patient showed negative Coomb's test and no haemoglobin or haemosiderin in urine. ${ }^{4}$ The increased reticulocyte count could be due to deficiency in iron and folate as well as vitamin $B_{12}$. Mild hyperbilirubinaemia usually occurs due to ineffective haemopoiesis resulting from folate and Vit-B-12 deficiency as it was probably true for our case. Iron and folate deficiency can easily be explained due to blood loss, dietary inadequacy and increase demand at the adolescent age. Our patient had only mild indirect hyperbilirubinaemia and no other evidence of organic liver disease. Her coagulation profile was normal, so disseminated intravascular coagulation was ruled out. Anti-platelet antibody could not be performed due to unavailability of the test though it lacks both sensitivity and specificity for diagnosis of ITP. Most specific therapeutic test to confirm the diagnosis of autoimmune nature of ITP is response to intravenous immunoglobulin, ${ }^{5}$ but our patient could not afford such a test. Prednisolone is the classical approach of treatment in ITP. This patient showed definite response with steroid.

This type of co-existent entities of multiple pathologies was not reported before in Bangladesh. It may be noted that presence of multiple pathologies, sometimes opposing in nature, may co-exist in a patient. Careful blood film examination by an experienced morphologist can guide investigations to the appropriate direction thus saving much time and money.

\section{References}

1. Lachant NA: Hemoglobin E, an emerging haemoglobinopathy in the Unites States. Am J Hematol 1987; 25: 449.

2. Shashik ant C.U. Patne, Jyoti Shukla. Hb-E disorders in Eastern Uttar Pradesh. Indian Journal of Pathology and Microbiology 2009; 52(1): 110-2.

3. Vichinsky E. Hemoglobin E Syndromes. Hematology 2007; 79-83.

4. Gordon-Smith EC and Marsh JCW: Acquired haemolytic anaemias. In: Hoffbrand AV, Catovsky D, Tuddenham EGD, editors. Postgraduate Haematology $5^{\text {th }}$ ed. 2005, Blackwell Publishing Ltd, Oxford, UK, 151-68.

5. Chiu A, Tam W, Cines D and Bussel JB. Immune thrombocytopenic purpura: pathophysiology in patients with persistent problems. In: Hoffbrand AV, Catovsky D, Tuddenham EGD, editors. Postgraduate Haematology $5^{\text {th }}$ ed. 2005, Blackwell Publishing Ltd, Oxford, UK, 937-44. 